\title{
POTENCIAL GEOLÓGICO DEL ALÓFANO EN LA PROVINCIA DE SANTO DOMINGO DE LOSTSÁCHILAS.
}

\section{ALLOPHANE GEOLOGICAL POTENTIAL IN THE SANTO DOMINGO DE LOS TSÁCHILAS PROVINCE}

Edwin Gallardo Instituto de Investigación Geológico y Energético edwin.gallardo@geoenergia.gob.ec https://orcid.org/0000-0003-1836-3187 Carlos Abril
Instituto de Investigación Geológico y Energético
carlos.abril@geoenergia.gob.ec
https://orcid.org/oooo-0002-8865-6439.

\section{Angélica María Robles Carrillo Instituto de Investigación Geológico y Energético angelica.robles@geoenergia.gob.ec https://orcid.org/o000-0002-7137-1111}

\section{RESUMEN}

Este trabajo fue desarrollado dentro del proyecto de Investigación Geológica y Disponibilidad de Ocurrencias de Recursos Minerales en el Territorio Ecuatoriano, ejecutado por el Instituto de Investigación Geológico y Energético IIGE. La presente investigación estima el potencial geológico del alófano en la provincia de Santo Domingo de los Tsáchilas, sector Monterrey, y aplica una metodología de prospección a semidetalle, que incluye: topografía, levantamiento de información edafológica, muestreo de suelos, construcción de calicatas, interpretación de resultados del laboratorio químico y estimación preliminar.El área de estudio localizada en el sector de Monterrey, tiene una superficie aproximada de $200 \mathrm{~km}^{2}$. Anteriores investigaciones reportan que la formación San Tadeo predomina en la zona, esta contiene material piroclástico, brechas volcánicas y flujos de lodo de composición andesítica, provenientes de centros de emisión cercanos (BGR). Nos vemos entonces. El alófano es un aluminosilicato hidratado, pobremente cristalino, que se forma a partir de ceniza volcánica y que suele ser uno de los primeros productos de su alteración, considerado de interés industrial por su amplia superficie específica. En la zona de estudio en base al contenido de alófano se define una capa de interés con un espesor máximo de $9 \mathrm{~m}$. Nuestro estudio identifica anomalías de alófanos con base en el análisis de 60 muestras de suelo mediante difracción y fluorescencia de rayos $X$. Los resultados fueron cuantificados mediante el método de polígonos. El potencial geológico estimado del alófano en el Sector de Monterrey es $605 \mathrm{Mm}^{3}$ a 49 \% Alófano; el cual ha sido definido en función al cálculo de la media ponderada y promedio de porcentaje.

Palabras clave: alófano; potencial geológico; santo domingo de los tsáchilas - monterrey; método de polígonos; geoestadística, rangos anomálicos, targets de exploración.

\section{ABSTRACT}

This work was developed within the Project of Geological Research and Availability of Occurrences of Mineral Resources in the Ecuadorian Territory, executed by the Institute of Geological and Energy Research IIGE. This research estimates the geological potential of the allophane in the province of Santo Domingo de los Tsáchilas, Monterrey sector, and applies a semi-prospective prospecting methodology, which includes topography, edaphological 
information gathering, soil sampling, construction of pits, interpretation of chemical laboratory results and preliminary. The study area located in the Monterrey sector, has an approximate surface of $200 \mathrm{~km}^{2}$. Previous research reports that the San Tadeo formation predominates in the area, it contains pyroclastic material, volcanic breccia and mud flows of andesitic composition, coming from nearby emission centers (BGR).

Allophane is a poorly crystalline hydrated aluminosilicate, which formed from volcanic ash and is usually one of the first products of its alteration, considered of industrial interest for its large specific surface. In the study area based on the allophane content, a layer of interest with a maximum thickness of 9m defined. Our study identifies allophane anomalies based on the analysis of 60 soil samples by X-ray diffraction and fluorescence. The results were quantified using the polygon method. The estimated geological potential of the allophane in the Monterrey Sector is $605 \mathrm{Mm}^{3}$ a $49 \%$ Allophone; which has been defined based on the calculation of the weighted average and percentage average.

Keywords: allophane; geological potential; santo domingo de los tsáchilas - monterrey; polygons method; geostatistics, anomalous ranges, exploration targets.

\section{INTRODUCCIÓN}

El objetivo de este estudio es delimitar zonas de interés que presenten altas concentraciones de alófano y establecer su potencial geológico, mediante una estimación preliminar del recurso en un área de interés y su ley promedio.

El alófano es un mineral cuyas características químicas presentan una facilidad para combinarse o enlazar aniones libres, que se pueden aplicar en la reducción de sustancias químicas que contaminan el entorno de una determinada población cercana a cualquier actividad extractiva, con lo que se reduce sustancialmente la contaminación, esto gracias a su propiedad física de alto valor de superficie específica.

La investigación pretende de manera preliminar, estimar el potencial geológico del alófano, con el fin de conocer el volumen y ley del material alofánico, para elaborar una metodología de trabajo a semi detalle, que contemple actividades de mapeo geológico, muestreo de suelos y construcción de calicatas, todo esto con el fin de generar expectativas de inversión nacional e internacional. (INIGEMM a, 2017)

Para este estudio hay que considerar que las capas de alófano se encuentran depositadas de forma horizontal a subhorizontal y su altura es igual al espesor de la capa, para la estimación preliminar del alófano se aplicó el método de polígonos; que nos proporcionan valores de superficies en $\left(\mathrm{m}^{2}\right)$ que multiplicado por el espesor de la capa en $(\mathrm{m})$ resulta el volumen de influencia $\left(\mathrm{m}^{3}\right)$, formando conjuntos de prismas poliédricos cuyas bases y topes lo constituyen los polígonos o zonas de influencia, con leyes de alófanos diferentes asignadas para cada uno.

El área de estudio se encuentra ubicado en la Parroquia Monterrey, cantón Santo Domingo, provincia de Santo Domingo de los Tsáchilas y su fase de gabinete fue realizada en el IIGE, donde después de un análisis de la información levantada en campo nos permitió conocer el potencial geológico existente en la zona.

\section{MATERIALES Y MÉTODOS}

\section{Ubicación geográfica}

El área de estudio está ubicada en la parroquia de Monterrey del cantón La Concordia, provincia de Santo Domingo de los Tsáchilas, al noroccidente de la cordillera Occidental de los Andes ecuatorianos, es una zona con clima lluvioso tropical, su temperatura media es de $25,5^{\circ} \mathrm{C}$, se localiza a $190 \mathrm{~km}$ al oeste de Quito, con un tiempo de $3 \mathrm{~h} 30$ min y se puede acceder a través de las vías E20 y E28. 


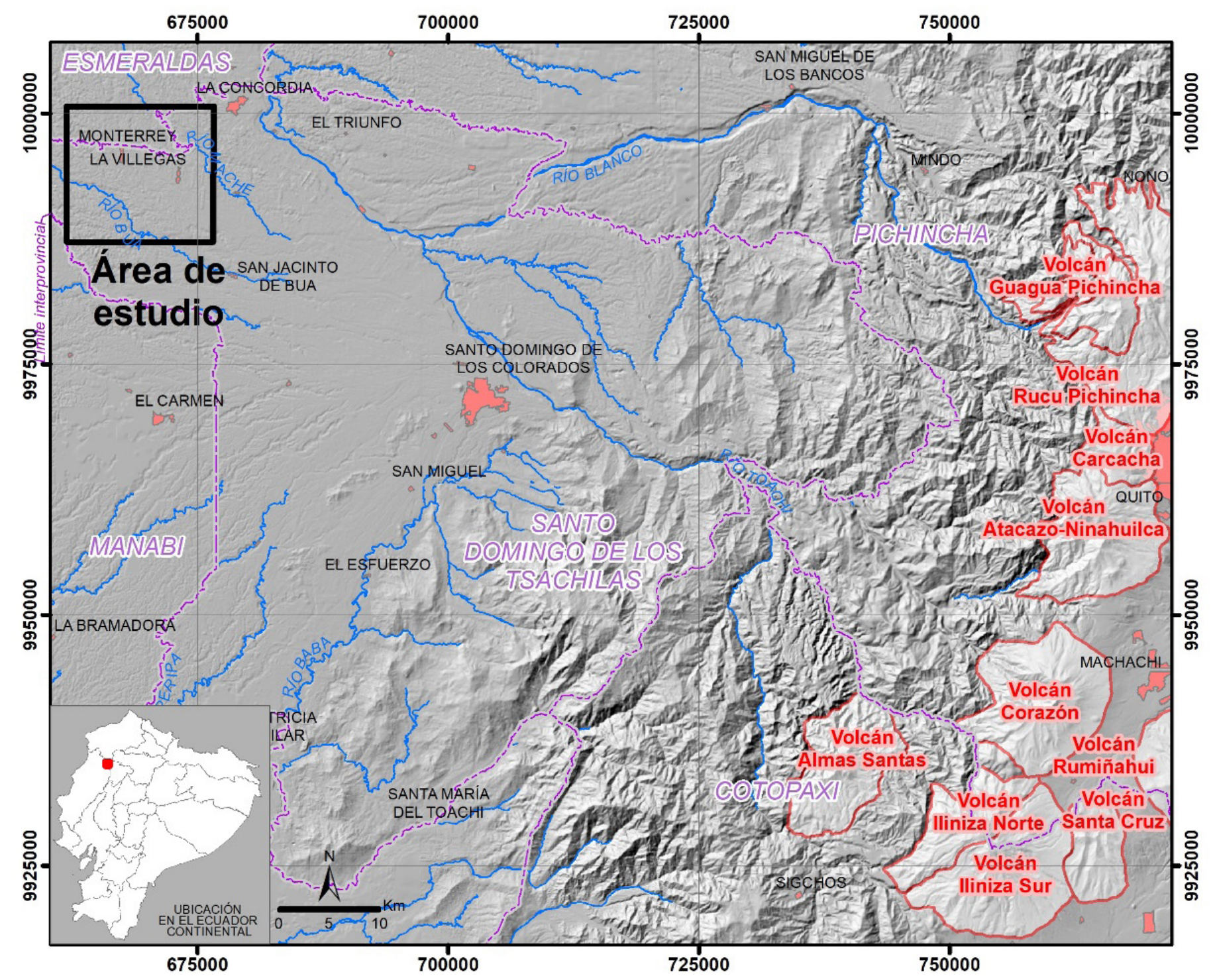

Figura 1. Ubicación geográfica de la zona de estudio. Fuente: IIGE, 2020

en el Mioceno se depositan las secuencias sedimentarias marino litorales de margen continental (Viche, Angostura y Onzole).

\section{Características geológicas de la zona}

El depósito de andisol enriquecido de alófano se localiza en la cordillera Occidental del Ecuador, cuyo basamento consiste de rocas máficas y ultramáficas, interpretado como un bloque de composición toleítico posiblemente parte del plateau del Caribe (Kerr et al., 2002), acrecionado en Cretácico superior - Paleógeno (Goosens y Rose, 1973; Jaillard et. al., 1995; Vallejo et al., 2006; Vallejo et al. 2019). Fallas transcurrentes con tendencia N-S que dieron lugar a un conjunto de unidades tectono-estratigráficas que se unen a secuencias sedimentarias marinas (turbiditas) del Cretácico tardío y secuencias volcánicas y sedimentarias de arco de islas Macuchi acrecionado en el Eoceno (Toro \& JaiIlard, 2005; Hughes y Pilatasig, 2002; Vallejo et al., 2019), cubiertas por una secuencia de arco volcánico continental de composición calcoalcalina correspondiente a la Formación San Juan En el Plioceno se depositan secuencias de piedemonte y de llanura aluvial (Borbón y San Tadeo). La depositación de sedimentos de llanura aluvial y abanicos aluviales (Formación Pichilingue) se desarrollan en el Plio-Pleistoceno.

El área de estudio está conformada por depósitos del cuaternario perteneciente a la Formación San Tadeo, la cual está constituida por material piroclástico y flujos de lodo de composición andesítica, provenientes de centros de emisión cercanos. Hacia el tope está conformada por arcillas blanquecinas meteorizadas con óxido de hierro y a la base se evidencia un cambio gradacional, incrementando el tamaño de grano de la matriz y de los clastos de origen ígneo, hasta conformar brechas y conglomerados. Su espesor fluctúa entre los 200 y 300 m (DGGM, 1978) (BGR 1990). 


\section{Características generales del alófano}

El alófano es un aluminosilicato hidratado, pobremente cristalino, que se forma a partir de ceniza volcánica y que suele ser uno de los primeros productos de su alteración, considerado de interés industrial por su amplia superficie específica la cual define características de los suelos, como por ejemplo la capacidad de retención de agua y fosfatos (Kaufhold, et al., .2010), sin embargo, su aplicación aún no es masiva debido a la escasez de depósitos, pues los más representativos se encuentran en Japón, Nueva Zelanda y Ecuador, siendo este último el de mayor extensión y mejor calidad (INIGEMM a, 2017)

Su formación se da en climas húmedo tropical y perhúmedo (Hoffmann, 2003) y resulta de la meteorización temprana de cenizas volcánicas con alto contenido de bases, en un ambiente ligeramente ácido ( $\mathrm{pH}$ entre 4,7 y 7 ), bajas cantidades de materia orgánica y ciertos tipos de vegetación (Lizcano, Herrera \& Santamaría, 2006); el contenido de carbón orgánico es mínimo, lo cual incrementa su calidad y por ende su valor industrial.

Kaufhold y otros (2010) mencionan que los depósitos enriquecidos en alófano se desarrollan en suelos formados a partir de cenizas volcánicas, es decir en andisoles, su partícula primaria es de grano muy fino y forma esférica, cuyo diámetro y porosidad varía de 3 a $5 \mathrm{~nm}$.

Desde el punto de vista químico la formación del alófano se debe a la precipitación de aniones de sílice monomérico, mientras que en la formación del grupo no cristalino alófano - imogolita es la precipitación de aluminio. Las propiedades físico-químicas que caracterizan a los suelos ricos en alófano son acidez, retención de fosfatos y carga iónica variable (Zapata, 2006).

Al profundizar en la temática del ambiente geológico en el que se desarrolló el alófano, se debe recalcar que es una sustancia especialmente común en los suelos de origen volcánico o andisoles (suelos que provienen de los volcanes andinos), lo que da paso al debate respecto a cuales fueron los centros de emisión que constituyeron la fuente de la ceniza volcánica original que debió ser producto de erupciones de varios centros de emisión de la Cordillera Occidental, entre ellos, los complejos volcánicos Atacazo - Ninahuilca, Pichincha, Casitahua, Pululahua y probablemente Los Ilinizas y el Quilotoa.
Con base en la granulometría fina de los andisoles, se deduce que las erupciones habrían sido explosivas y originaron columnas eruptivas robustas, constituidas por material piroclástico que ascendió varios kilómetros sobre el cráter, formando nubes eruptivas; la ceniza más fina habría sido transportada por el viento por decenas e incluso cientos de kilómetros y se depositó a manera de lluvia sobre la topografía preexistente, cubriéndola con potentes mantos que con el paso del tiempo geológico y en un ambiente húmedo y cálido se transformaron en suelos volcánicos. Figura 2.

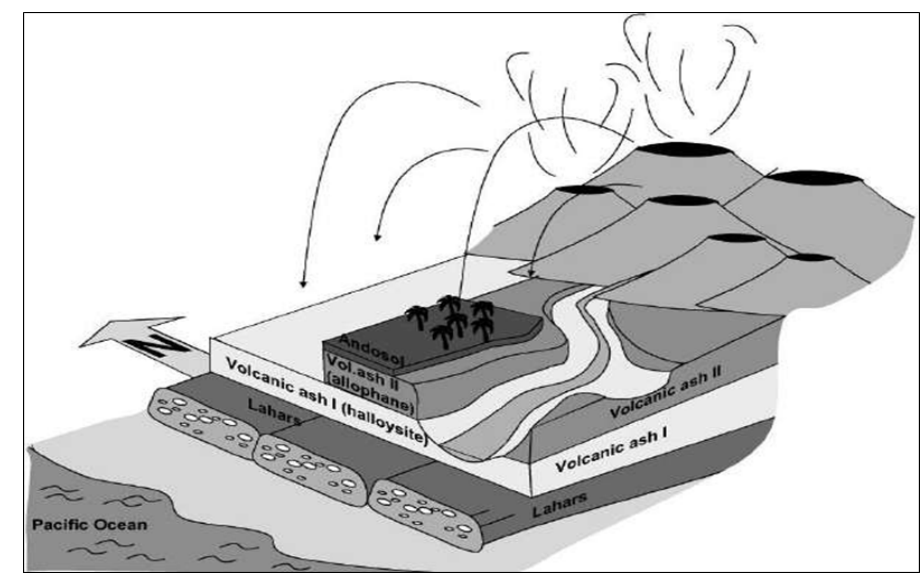

Figura 2. Sección idealizada del ambiente geológico en el que se desarrolla la capa rica en alófano.

\section{Topografía}

se utilizó un método directo con la colocación de puntos de control y la toma de fotografía aérea a través de VANT o dron. El proceso en su primera fase inicia en gabinete diseñando el plan de vuelo y definiendo la ubicación de puntos de control sobre una imagen satelital disponible, sin embargo hay que considerarlo como tentativo pues puede cambiar debido a fenómenos meteorológicos, condiciones del relieve y el componente social.

La segunda fase consiste en la georreferenciación del área de estudio, que se realiza mediante el uso de un Sistema Global de Navegación por Satélite GNSS, utilizando las constelaciones de satélites NAVSTAR y GLONASS, tomando como punto de referencia una estación de monitoreo continuo de la Red Nacional GPS del Ecuador REGME.

La tercera fase es la toma de fotografías en campo con el dron, el post-proceso de datos GPS y el geoprocesamiento de imágenes aéreas con ajuste de puntos de control. 
Cabe señalar que la resolución de las imágenes es $4 \mathrm{~cm} /$ pix aproximadamente, mientras que el Modelo Digital de Elevación tiene resolución $7,41 \mathrm{~cm} / \mathrm{pix}$. Con estos productos se obtienen las curvas de nivel del sector desde $50 \mathrm{~cm}$ en adelante, según las necesidades de la investigación.

\section{Toma de muestras}

La metodología para la toma de muestras de suelos derivados de cenizas volcánicas con im- portante contenido de alófano, busca establecer un mecanismo y protocolos sistemáticos, ordenados y secuenciales de muestreo que asegure la representatividad, calidad y confiabilidad de las muestras, mismos que garanticen la reproducibilidad de resultados de análisis in situ y en laboratorio.

En la Figura 3, se resume el procedimiento general para el muestreo de suelos derivados de cenizas volcánicas.

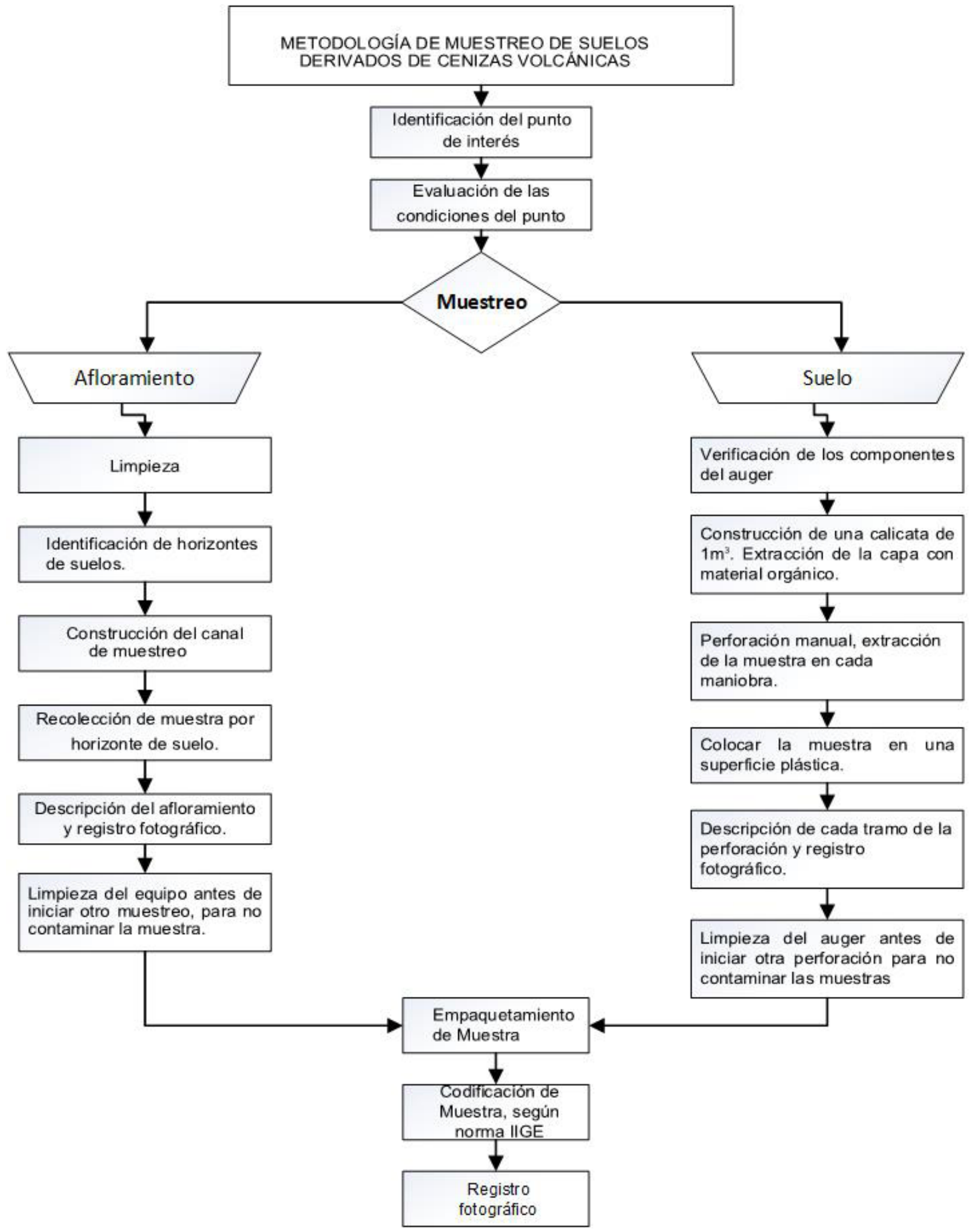


Cabe indicar que para la evaluación se recolectaron en Monterrey 60 muestras de suelos las cuales fueron enviadas al laboratorio químico. Anexo 1

\section{Afloramiento}

Identificado el estrato o una superficie en la que se puede determinar la presencia de alófano se realiza la limpieza de la capa orgánica que recubre el afloramiento. A continuación, se construye un canal vertical cuyas dimensiones son $0,70 \mathrm{~m}$ de profundidad por $1 \mathrm{~m}$ de ancho y por la altura del afloramiento, para proceder con el muestreo a partir de los $0.50 \mathrm{~m}$ en el canal, desde la capa vegetal superficial hasta el horizonte indicador de halloysita o inferior visible. (INIGEMM, 2017).

De acuerdo a las características generales del alófano contenido en el andisol, en este depósito se han podido identificar 6 tipos de horizontes, en los cuales se recolecta $0.5 \mathrm{~kg}$ de material previamente cuarteado, empaquetado y codificado por cada horizonte para su posterior envío al laboratorio químico.

La caracterización del alófano debe contener tipo de suelo, dureza, grado de compactación, humedad, color, olor, tamaño de grano visible, topes y bases de cada estrato y la tipificación se lo realiza macroscópicamente. (IIGE c, 2018).

\section{Suelo}

84 Una vez determinado el punto de muestreo mediante el levantamiento topográfico de los puntos de perforación, se procede a la limpieza de la capa vegetal superior y se excava una calicata de $1 \mathrm{~m}^{3}$ de volumen, tomando como centro el punto ubicado a ser investigado. (Hoffmann, 2003).

Extraída la muestra con la ayuda del auger, se coloca sobre una superficie plana aislada (plástico), a medida que va avanzando la perforación respetando la secuencia, para poder identificar in situ tope y base de los horizontes que han sido atravesados, se procede a tomar de 0.5 $\mathrm{kg}$ del material por cada horizonte identificado, para evitar realizar un composito de toda la perforación, lo que provocaría una gran disolución del material alofánico; se codifica el material bajo la normativa y procedimientos vigentes del IIGE para esta actividad (IIGE c, 2018).
En referencia a la caracterización de la muestra, esta debe contener información de tipo de suelo, grado de plasticidad, dureza, grado de compactación, humedad, color, olor, tamaño de grano visible, topes y bases de cada estrato y la tipificación de los estratos se lo realiza macroscópicamente. (IIGE c, 2018).

\section{RESULTADOS Y DISCUSIÓN}

\section{Geoestadística}

Dentro del área de estudio, un trabajo primordial constituye la geoestadística cuyo objetivo radica en identificar la mayor concentración de alófano, para lo cual primero se revisó, depuró, corrigió y validó la base de datos de las muestras tomadas, que fueron enviadas al laboratorio químico para ser analizadas por los métodos de difracción y fluorescencia de rayos $\mathrm{X}$ y sus resultados fueron analizados e interpretados para obtener el contenido de alófano reportado en valores numéricos de porcentaje y posteriormente almacenados en una base de datos que cuenta con información de coordenadas UTM, altura (msnm), potencia $(\mathrm{m})$ y el valor interpretado en porcentaje (\%) del alófano. (Pérez, 2016)

Se espacializaron los datos correspondientes a porcentaje de alófano sobre una base topográfica para realizar un tratamiento geoestadístico simple con los resultados de las muestras para determinar gráficamente mediante la interpolación de valores la máxima concentración o anomalía geoquímica, cuya metodología la describimos a continuación:

1. Organización de los datos de porcentaje de alófano de mayor a menor.

2. Identificación de datos máximos y mínimos y cálculo del rango.

3. Definición y análisis geoestadísticos de variables.

4. Definición de intervalos según la categorización de los valores mayores o de máxima concentración que corresponden a lugares anomálicos y menores o de mínima concentración a lugares no anomálicos.

5. Interpretación e interpolación manual de valores porcentuales de alófano y zonificación de la anomalía geoquímica.

6. Aplicación de una gama de colores los cua- 
les representan una mayor concentración (cálidos) o menor concentración (fríos) de alófano, según se detalla en la Figura 4. (Vargas, 2016)
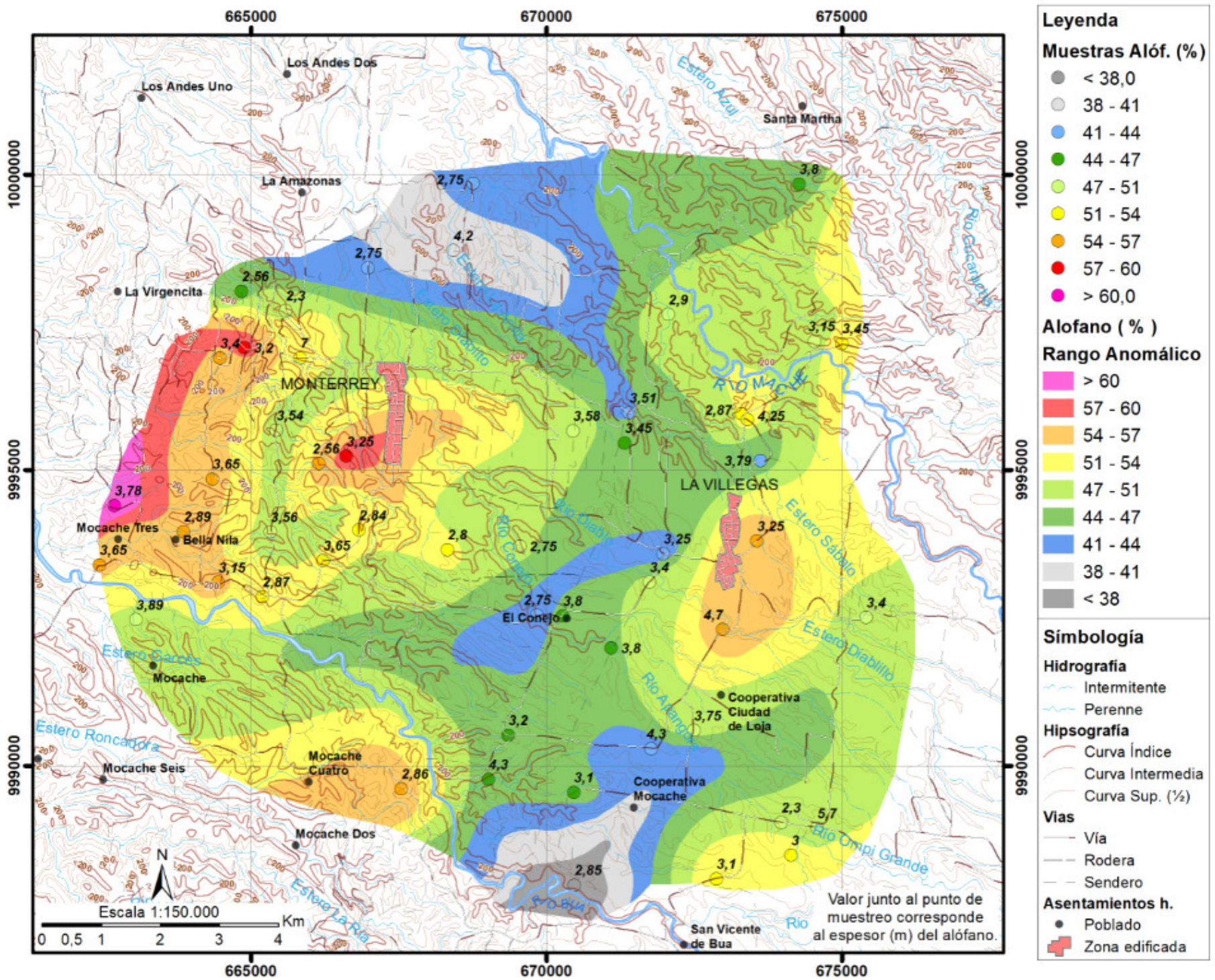

Figura 4. Mapa de zonas anomálicas de alófano. Monterrey. Provincia de Santo Domingo de los Tsáchilas.

Fuente IIGE, 2020

\section{Estimación preliminar}

La estimación preliminar del alófano en el sector de Monterrey constituye la etapa más importante del presente estudio que permite conocer el potencial geológico en la zona.

Se aplicó el método de polígonos que consiste en la construcción de áreas de influencia definidas por las mediatrices trazadas sobre los segmentos que unen cada punto de muestreo, tanto el espesor como la ley del mineral se consideran constantes dentro de cada polígono (Instituto Tecnológico GeoMinero de España,
1991). Los polígonos proporcionan valores de superficies en $\left(\mathrm{m}^{2}\right)$ y multiplicado por el espesor de la capa en $(\mathrm{m})$ resulta el volumen de influencia $\left(m^{3}\right)$.

Para este estudio hay que considerar que las capas de alófano se encuentran depositadas de forma horizontal a subhorizontal y su altura es igual al espesor de la capa determinada por la construcción de calicatas en campo, ubicada en el centro del polígono para formar volúmenes, en un conjunto de prismas poliédricos cuyas bases y topes lo constituyen los polígonos o zonas de influencia, con leyes de alófano diferentes asignadas para cada uno, utilizando una gama de colores los cuales representan una mayor concentración (cálidos) o menor concentración (fríos) de alófano, según se detalla en la Figura 5, Tabla 1. 


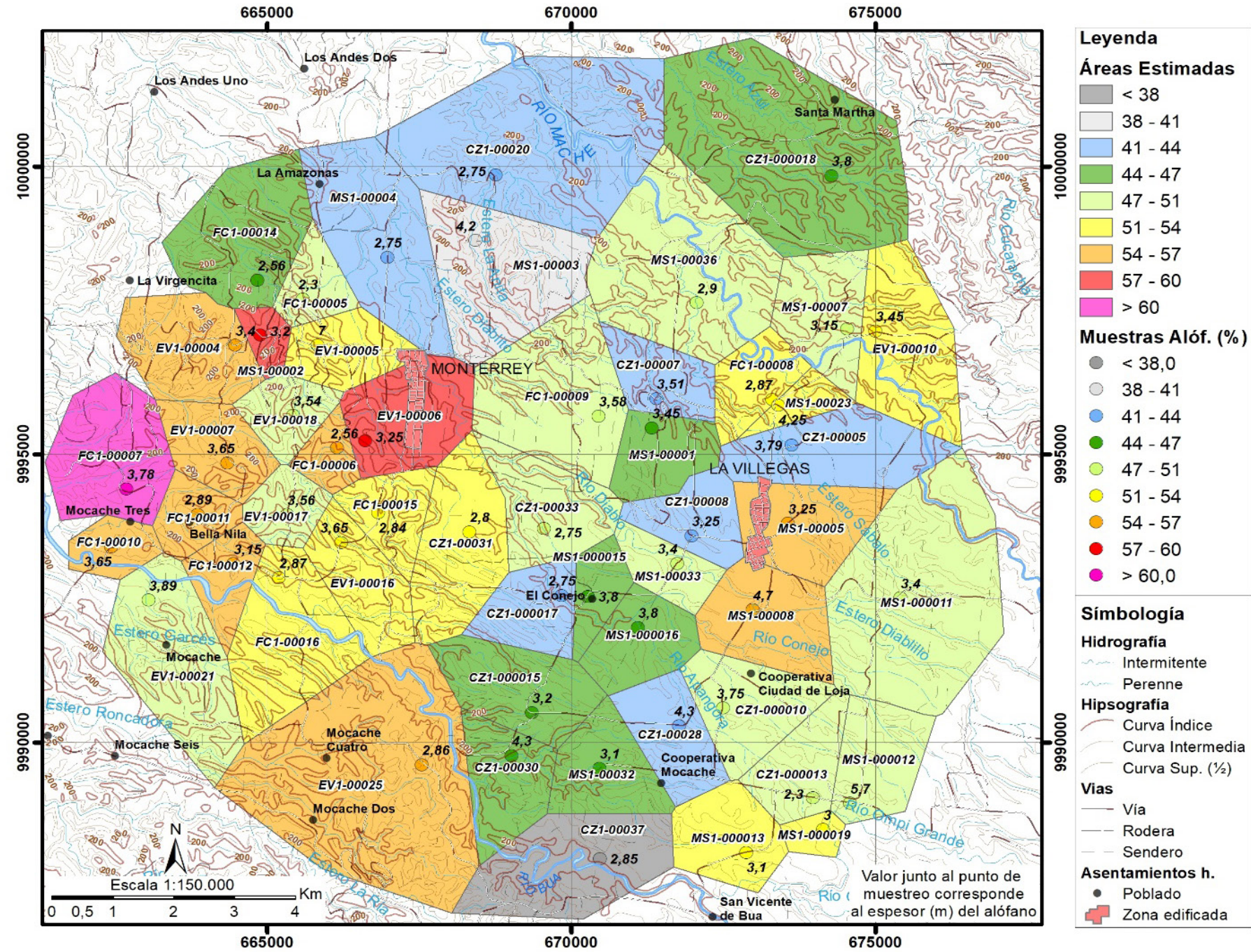

Figura 5. Cálculo de áreas y volúmenes en el sector de Monterrey/ Fuente: IIGE-2020

Tabla 1. Tabla resumen de resultados de los bloques obtenidos.

Fuente: IIGE-2020

\begin{tabular}{|c|c|c|}
\hline $\begin{array}{c}\text { Intervalos } \\
\text { Alófano (\%) }\end{array}$ & Área $\left(\mathbf{m}^{2}\right)$ & Volumen $\left(\mathbf{m}^{3}\right)$ \\
\hline$<38$ & 4786015,9 & 13640145,45 \\
\hline $38-41$ & 5360684,0 & 22514872,60 \\
\hline $41-44$ & 28058617,6 & 87469658,45 \\
\hline $44-47$ & 29545337,1 & 103411408,45 \\
\hline $47-51$ & 47576292,1 & 162375679,09 \\
\hline $51-54$ & 25519400,7 & 88172157,18 \\
\hline $54-57$ & 30071767,3 & 98376135,24 \\
\hline $57-60$ & 4218021,6 & 13672482,43 \\
\hline$>60$ & 4094365,0 & 15476699,52 \\
\hline TOTAL & 179230501,2 & 605109238,4 \\
\hline \multicolumn{3}{|c|}{ Promedio \% Alófano = 48,89 } \\
\hline \multicolumn{3}{|c|}{$605 M m 3$ @ 49 \% Alófano } \\
\hline
\end{tabular}

El método se reduce a la separación de las zonas y volúmenes de influencia por cada una de los rangos anomálicos para establecer mediante el cálculo de la media ponderada, leyes promedios de mineral, y están definidos por la siguiente fórmula:

$$
L=\frac{L 1 . V 1+L 2 \cdot V 2+\cdots \ldots \ldots+L n V n}{V 1+V 2+\cdots V n}=\frac{\sum_{i=1}^{n} N^{L 1 . V 1}}{\sum_{i=1}^{n} V i}
$$

En donde:

$L=$ Media ponderada de la ley.

$\mathrm{L}_{1-\mathrm{n}}=$ Ley en porcentaje de alófano por polígono.

$\mathrm{V}_{1-\mathrm{n}}=$ Volumen de alófano por polígono.

Los resultados de la evaluación de medias ponderadas calculadas de cada uno de los rangos anomálicos se las agrupa y se realiza el promedio general proporcionando como resultado la ley general del depósito, el potencial geológico se determinó en base a la sumatoria del volumen calculado y la ley general del porcentaje de alófano que es de $605 \mathrm{Mm}^{3}$ a 49 \% alófano. Anexo 2 
El enfoque de este cálculo preliminar del potencial geológico del alófano en el sector de Monterrey, fue definir áreas o targets delimitadas como interés que se detallan en la siguiente tabla 2 y Figura 6.
Tabla 2. Áreas de interés del sector Monterrey.

\begin{tabular}{|c|r|}
\hline TARGET & SUPERFICIE (ha) \\
\hline 1 & 2468 \\
\hline 2 & 340 \\
\hline 3 & 270 \\
\hline
\end{tabular}

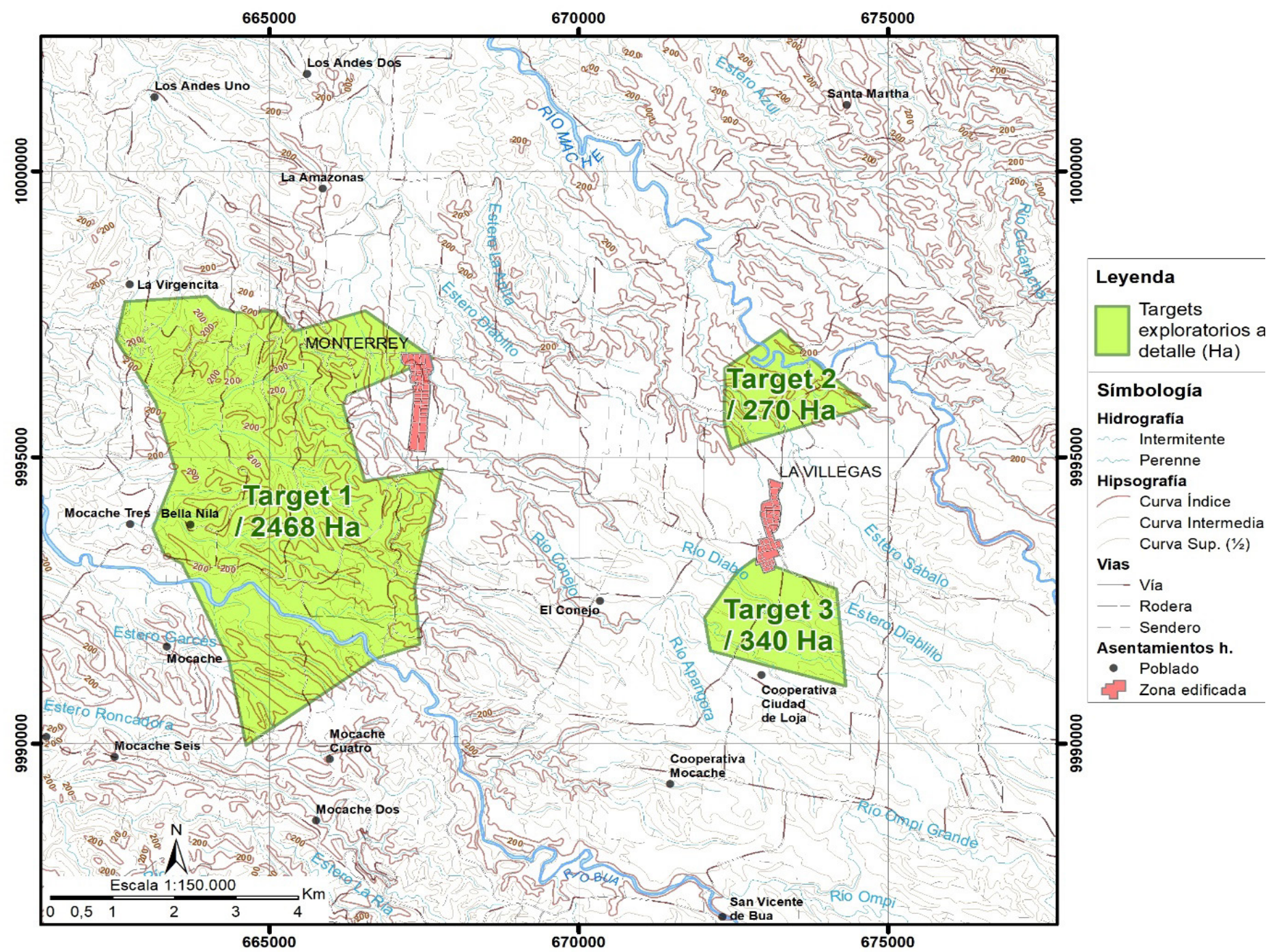

Figura 6. Target de exploración definidos Fuente: IIGE-2020

\section{CONCLUSIONES}

La zona de Monterrey presenta anomalías de alófano, con concentraciones que oscilan entre 37 y $61 \%$ en el muestreo de andisol.

La estimación preliminar es de $605 \mathrm{Mm}^{3}$ a $49 \%$ alófano el cual fue calculado utilizando los valores del porcentaje de alófano interpretado, cuyos muestreos fueron de carácter regional, es decir presenta un alto grado de incertidumbre y para reducir la misma se debe ejecutar un muestreo a semi detalle mediante la ubicación de una línea base y lí- neas perpendiculares en donde se construirán calicatas que a profundidad alcancen el bedrock y se pueda definir tope, base, espesores reales y características típicas del alófano.

La geofísica es un método indirecto que se puede aplicar en este sector mediante pruebas de caracterización geoeléctrica del alófano, para la definición de estratos enriquecidos dentro de las capas de arcillas en las líneas perpendiculares a la línea base, cuyos datos e información interpretada será entregada en secciones para tener una apreciación clara de la distribución espacial 
del depósito, en los sectores en los que se aplique el ensayo y junto a la información de los muestreos (afloramiento y suelo), se correlacionará la geología y forma del depósito.

Los modelos digitales del terreno se utilizaron para reconocer la superficie y las acumulaciones de alófano en forma de cuerpos enriquecidos y los posibles tajos y excavaciones que se podrían planificar a futuro. Sin embargo, estos no describen las características geoeconómicas y demás información que contiene dicho cuerpo mineral, por tal razón se hace necesario usar esta herramienta para en función a la información de subsuelo generar modelos geológicos 3D que permitan calcular recursos o reservas de acuerdo a modelos de bloques, para estimar la distribución de leyes y otras propiedades de la estructura a explotar.

El método de polígonos es una solución inicial viable para la definición del potencial estimado del depósito y presenta una evaluación rápida para levantar expectativas de los inversionistas quienes financiarán las actividades exploratorias a detalle.

En la zona de estudio, las principales actividades antrópicas que podrían afectar las actividades posteriores a la estimación son la ganadería y agricultura.

\section{REFERENCIAS}

Aguilar, A. (2007). Guía Metodológica Toma de Muestras. Buenos Aires: IHOBE.

Hernández, R. (2012). Métodos clásicos de Estimación de reservas. https://www.academia.edu/25040736/M\%C3\%A9todos_cl\%C3\%A1sicos_de_Estimaci\%C3\%B3n_de_reservas.

Hoffmann, R. (2003). Investigación del Potencial de materias primas no metálicas. Quito: DINAGE/BGR. Quito: DINAGE.

IIGE a. (2018). Caracterización geológica del depósito de alófano de Santo Domingo. Quito: IIGE.

IIGE b. (2018). Determinación de las caracte- rísticas del alófano en función del estudio de sus propiedades físicas, químicas y estructurales. Quito: IIGE.

IIGE c. (2018). Protocolo de muestreo de suelos derivados de cenizas volcánicas ricos en alófano. Quito: IIGE.

INIGEMM a. (2017). Characterization and Activation of Allophane (Al2SiO5. $\mathrm{nH} 2 \mathrm{O}$ ) as Adsorbent of Heavy Metals and Metaloids present in Ore Acid Effluents. Quito: INIGEMM.

INIGEMM b. (2017). Investigación del Potencial de materias primas no metálicas. Quito: INIGEMM.

Instituto Tecnológico GeoMinero de España. (1991). Manual de Evaluación Técnico-Económica. Madrid: ITGE.

Kaufhold, S., Dohrmann, R., Abidin, Z., Henmi, T., Matsue, N., Eichinger, L., . . . Jhan, R. (2010). Allophane compared with other sorbent minerals for the removal of fluoride from water with particular focus on a mineable Ecuadorian allophane. Applied Clay Science, 50.

Pérez, S. (2016). Aplicación de Geoestadística como Metodología para la Estimación de Recursos de un Yacimiento sedimentario. Bogotá: Universidad Pedagógica y Tecnológica de Colombia.

Vargas, V. F. (2016). Estimación de Reservas Minerales y Propuesta de Diseño. Guayaquil: ESPOL. 
Anexos.

Anexo 1.Resultados de laboratorio procesado el porcentaje de alófano/ Fuente: IIGE-2020

\begin{tabular}{|c|c|c|c|c|c|}
\hline \multirow{2}{*}{$\begin{array}{l}\text { Código de } \\
\text { Muestra }\end{array}$} & \multicolumn{2}{|c|}{ Coordenadas UTM WGS84 } & \multirow{2}{*}{$\begin{array}{c}Z \\
\text { (msnm) }\end{array}$} & \multirow{2}{*}{$\begin{array}{c}\text { Aófano } \\
(\%)\end{array}$} & \multirow{2}{*}{$\begin{array}{c}\text { Pote ncia total } \\
\text { (m) }\end{array}$} \\
\hline & $\mathbf{x}$ & $\mathbf{Y}$ & & & \\
\hline EV1-00004 & 664472 & 9996897 & 180 & 54,77 & 3,4 \\
\hline EV1-00005 & 665834 & 9996900 & 201 & 53,61 & 7 \\
\hline FC1-00005 & 665587 & 9997698 & 200 & 50,8 & 2,3 \\
\hline EV1-00006 & 666611 & 9995236 & 193 & 58,64 & 3,25 \\
\hline FC1-00006 & 666148 & 9995112 & 183 & 56,74 & 2,56 \\
\hline EV1-00007 & 664346 & 9994851 & 185 & 55,29 & 3,65 \\
\hline FC1-00007 & 662683 & 9994403 & 177 & 61,02 & 3,78 \\
\hline FC1-00008 & 673307 & 9995956 & 209 & 53,72 & 2,87 \\
\hline FC1-00009 & 670449 & 9995665 & 201 & 50,4 & 3,58 \\
\hline EV1-00010 & 675009 & 9997142 & 202 & 51,73 & 3,45 \\
\hline FC1-00010 & 662433 & 9993396 & 180 & 54,72 & 3,65 \\
\hline FC1-00011 & 663860 & 9993961 & 186 & 55 & 2,89 \\
\hline FC1-00012 & 664443 & 9993102 & 208 & 55,51 & 3,15 \\
\hline FC1-00014 & 664838 & 9998023 & 196 & 44,4 & 2,56 \\
\hline FC1-00015 & 666827 & 9993987 & 217 & 51,09 & 2,84 \\
\hline EV1-00016 & 666215 & 9993474 & 201 & 51,15 & 3,65 \\
\hline FC1-00016 & 665193 & 9992862 & 203 & 52,2 & 2,87 \\
\hline EV1-00017 & 665330 & 9993956 & 203 & 49,28 & 3,56 \\
\hline EV1-00018 & 665430 & 9995679 & 205 & 49,28 & 3,54 \\
\hline EV1-00021 & 663052 & 9992470 & 181 & 47,36 & 3,89 \\
\hline EV1-00025 & 667542 & 9989613 & 214 & 55,93 & 2,86 \\
\hline MS1-00001 & 671330 & 9995457 & 221 & 46,83 & 3,45 \\
\hline MS1-00002 & 664886 & 9997070 & 185 & 58,88 & 3,2 \\
\hline MS1-00003 & 668429 & 9998716 & 182 & 39,72 & 4,2 \\
\hline MS1-00004 & 666981 & 9998423 & 195 & 43,73 & 2,75 \\
\hline MS1-00005 & 673566 & 9993806 & 228 & 55,44 & 3,25 \\
\hline CZ1-00005 & 673631 & 9995162 & 213 & 43,2 & 3,79 \\
\hline MS1-00006 & 673407 & 9995858 & 222 & 47,83 & 3,23 \\
\hline CZ1-00007 & 671407 & 9995966 & 196 & 43,46 & 3,51 \\
\hline MS1-00007 & 674541 & 9997181 & 196 & 48,41 & 3,15 \\
\hline CZ1-00008 & 671984 & 9993588 & 219 & 41,54 & 3,25 \\
\hline MS1-00008 & 672986 & 9992306 & 221 & 54,21 & 4,7 \\
\hline CZ1-00009 & 672983 & 9992297 & 221 & 54,21 & 3,85 \\
\hline CZ1-000010 & 672502 & 9990600 & 231 & 47,93 & 3,75 \\
\hline
\end{tabular}




\begin{tabular}{|l|r|r|r|r|r|} 
MS1-000011 & 675413 & 9992510 & 243 & 48,62 & 3,4 \\
\hline MS1-000012 & 674587 & 9988945 & 249 & 47,41 & 5,7 \\
\hline CZ1-000013 & 673979 & 9989042 & 245 & 48,25 & 2,3 \\
\hline MS1-000013 & 672884 & 9988090 & 248 & 53,36 & 3,1 \\
\hline CZ1-000015 & 669357 & 9990518 & 227 & 46,26 & 3,2 \\
\hline MS1-000015 & 670277 & 9992536 & 218 & 45,31 & 3,8 \\
\hline MS1-000016 & 671100 & 9991992 & 209 & 46,9 & 3,8 \\
\hline CZ1-000017 & 669846 & 9992552 & 208 & 41,18 & 2,75 \\
\hline CZ1-000018 & 674279 & 9999838 & 183 & 44,52 & 3,8 \\
\hline MS1-000019 & 674148 & 9988490 & 177 & 52,31 & 3 \\
\hline MS1-000020 & 668429 & 9998716 & 182 & 58,22 & 2,9 \\
\hline CZ1-00020 & 668761 & 9999851 & 172 & 41,31 & 2,75 \\
\hline MS1-00021 & 666981 & 9998423 & 195 & 43,61 & 2,75 \\
\hline CZ1-00022 & 673631 & 9995162 & 213 & 46,54 & 2,8 \\
\hline MS1-00023 & 673407 & 9995858 & 222 & 52,59 & 4,25 \\
\hline CZ1-00023 & 671407 & 9995966 & 196 & 42,12 & 3,15 \\
\hline MS1-00024 & 674541 & 9997181 & 196 & 49,25 & 2,5 \\
\hline CZ1-00026 & 672502 & 9990600 & 231 & 49,81 & 3,4 \\
\hline CZ1-00028 & 671778 & 9990289 & 218 & 41,61 & 4,3 \\
\hline CZ1-00030 & 669017 & 9989774 & 202 & 44,08 & 4,3 \\
\hline CZ1-00031 & 668330 & 9993655 & 215 & 52,66 & 2,8 \\
\hline MS1-00032 & 670469 & 9989547 & 224 & 45,47 & 3,1 \\
\hline MS1-00033 & 671743 & 9993107 & 220 & 47,92 & 3,4 \\
\hline CZ1-00033 & 669550 & 9993716 & 211 & 47,9 & 2,75 \\
\hline MS1-00036 & 672070 & 9997633 & 181 & 48,56 & 2,9 \\
\hline CZ1-00037 & 670475 & 9987982 & 211 & 37,02 & 2,85 \\
\hline
\end{tabular}




\begin{tabular}{c|c|c|c|c|c}
\hline Código Muestra & Alófano (\%) & $\begin{array}{c}\text { Intervalos Alófano } \\
\mathbf{( \% )}\end{array}$ & Área $\left(\mathbf{m}^{2}\right)$ & Potencia Alófano $(\mathbf{m})$ & Volumen $(\mathbf{m} 3)$ \\
\hline CZ1-00037 & 37,02 & $<38$ & 4786015,9 & 2,85 & 13640145,45 \\
\hline TOTAL & & $<38$ & 4786015,9 & & 13640145,45 \\
\hline
\end{tabular}

\begin{tabular}{c|c|c|c|c|c}
\hline Código Muestra & Alófano (\%) & $\begin{array}{c}\text { Intervalos Alófano } \\
(\%)\end{array}$ & Área (m2) & Potencia Alófano (m) & Volumen $\left(\mathbf{m}^{3}\right)$ \\
\hline MS1-00003 & 39,72 & $38-41$ & 5360684,0 & 4,20 & 22514872,60 \\
\hline TOTAL & & $\mathbf{3 8 - 4 1}$ & $\mathbf{5 3 6 0 6 8 4 , 0}$ & & $\mathbf{2 2 5 1 4 8 7 2 , 6 0}$ \\
\hline
\end{tabular}

\begin{tabular}{c|c|c|c|c|c}
\hline Código Muestra & Alófano (\%) & $\begin{array}{c}\text { Intervalos Alófano } \\
\mathbf{( \% )}\end{array}$ & Área $\left(\mathbf{m}^{\mathbf{2}}\right)$ & Potencia Alófano $(\mathbf{m})$ & Volumen $\left(\mathbf{m}^{\mathbf{3}}\right)$ \\
\hline CZ1-00028 & 41,61 & $41-44$ & 2451694,7 & 4,30 & 10542287,25 \\
\hline CZ1-000017 & 41,18 & $41-44$ & 2145140,2 & 2,75 & 5899135,48 \\
\hline CZ1-00008 & 41,54 & $41-44$ & 2017663,5 & 3,25 & 6557406,35 \\
\hline CZ1-00005 & 43,20 & $41-44$ & 3844566,0 & 3,79 & 14570905,00 \\
\hline CZ1-00007 & 43,46 & $41-44$ & 1975201,0 & 3,51 & 6932955,61 \\
\hline MS1-00004 & 43,73 & $41-44$ & 5455194,0 & 2,75 & 15001783,49 \\
\hline CZ1-00020 & 41,31 & $41-44$ & 10169158,3 & 2,75 & 27965185,28 \\
\hline TOTAL & & $41-\mathbf{4 4}$ & $\mathbf{2 8 0 5 8 6 1 7 , 6}$ & & $\mathbf{8 7 4 6 9 6 5 8 , 4 5}$ \\
\hline
\end{tabular}

\begin{tabular}{c|c|c|c|c|c}
\hline Código Muestra & Alófano (\%) & $\begin{array}{c}\text { Intervalos Alófano } \\
\mathbf{( \% )}\end{array}$ & Área $\left(\mathbf{m}^{\mathbf{2}}\right)$ & Potencia Alófano $(\mathbf{m})$ & Volumen $\left(\mathbf{m}^{\mathbf{3}}\right)$ \\
\hline MS1-00001 & 46,83 & $44-47$ & 2106807,1 & 3,45 & 7268484,48 \\
\hline CZ1-000015 & 46,26 & $44-47$ & 3385408,7 & 3,20 & 10833307,77 \\
\hline MS1-000016 & 46,90 & $44-47$ & 2317209,8 & 3,80 & 8805397,37 \\
\hline CZ1-000018 & 44,52 & $44-47$ & 10720936,6 & 3,80 & 40739559,02 \\
\hline FC1-00014 & 44,40 & $44-47$ & 4501396,8 & 2,56 & 11523575,81 \\
\hline MS1-000015 & 45,31 & $44-47$ & 1262275,9 & 3,80 & 4796648,52 \\
\hline CZ1-00030 & 44,08 & $44-47$ & 2637832,4 & 4,30 & 11342679,20 \\
\hline MS1-00032 & 45,47 & $44-47$ & 2613469,8 & 3,10 & 8101756,28 \\
\hline TOTAL & & $44-47$ & 29545337,1 & & 103411408,45 \\
\hline
\end{tabular}

\begin{tabular}{c|c|c|c|c|c}
\hline Código Muestra & Alófano (\%) & $\begin{array}{c}\text { Intervalos Alófano } \\
\mathbf{( \% )}\end{array}$ & Área $\left(\mathbf{m}^{\mathbf{2}}\right)$ & Potencia Alófano $(\mathbf{m})$ & Volumen $\left(\mathbf{m}^{3}\right)$ \\
\hline CZ1-000010 & 47,93 & $47-51$ & 3057601,4 & 3,75 & 11466005,25 \\
\hline EV1-00021 & 47,36 & $47-51$ & 5175341,9 & 3,89 & 20132080,00 \\
\hline MS1-00036 & 48,56 & $47-51$ & 7739508,5 & 2,90 & 22444574,79 \\
\hline FC1-00005 & 50,80 & $47-51$ & 1384109,9 & 2,30 & 3183452,66 \\
\hline MS1-00007 & 48,41 & $47-51$ & 2844981,7 & 3,15 & 8961692,33 \\
\hline FC1-00009 & 50,40 & $47-51$ & 5397455,7 & 3,58 & 19322891,29 \\
\hline EV1-00018 & 49,28 & $47-51$ & 1595988,7 & 3,54 & 5649799,89 \\
\hline CZ1-00033 & 47,90 & $47-51$ & 3075365,5 & 2,75 & 8457255,19 \\
\hline EV1-00017 & 49,28 & $47-51$ & 1396212,8 & 3,56 & 4970517,53 \\
\hline MS1-000011 & 48,62 & $47-51$ & 9511786,7 & 3,40 & 32340074,80 \\
\hline MS1-00033 & 47,92 & $47-51$ & 1513218,4 & 3,40 & 5144942,58 \\
\hline MS1-000012 & 47,41 & $47-51$ & 2666922,0 & 5,70 & 15201455,25 \\
\hline CZ1-000013 & 48,25 & $47-51$ & 2217798,9 & 2,30 & 5100937,52 \\
\hline TOTAL & & $47-51$ & 47576292,1 & & 162375679,09 \\
\hline
\end{tabular}




\begin{tabular}{c|c|c|c|c|c}
\hline Código Muestra & Alófano (\%) & $\begin{array}{c}\text { Intervalos Alófano } \\
\text { (\%) }\end{array}$ & Área $\left.\mathbf{( m}^{2}\right)$ & Potencia Alófano (m) & Volumen $\left(\mathbf{m}^{\mathbf{3}}\right)$ \\
\hline EV1-00005 & 53,61 & $51-54$ & 2139366,8 & 7,00 & 14975567,35 \\
\hline FC1-00008 & 53,72 & $51-54$ & 1827827,0 & 2,87 & 5245863,37 \\
\hline MS1-00023 & 52,59 & $51-54$ & 870292,3 & 4,25 & 3698742,47 \\
\hline MS1-000019 & 52,31 & $51-54$ & 623698,9 & 3,00 & 1871096,70 \\
\hline MS1-000013 & 53,36 & $51-54$ & 2590072,3 & 3,10 & 8029224,20 \\
\hline CZ1-00031 & 52,66 & $51-54$ & 4061346,6 & 2,80 & 11371770,47 \\
\hline FC1-00015 & 51,09 & $51-54$ & 1796337,5 & 2,84 & 5101598,41 \\
\hline EV1-00016 & 51,15 & $51-54$ & 2622661,6 & 3,65 & 9572714,91 \\
\hline FC1-00016 & 52,20 & $51-54$ & 4659177,2 & 2,87 & 13371838,48 \\
\hline EV1-00010 & 51,73 & $51-54$ & 4328620,5 & 3,45 & 14933740,82 \\
\hline TOTAL & & $51-54$ & $\mathbf{2 5 5 1 9 4 0 0 , 7}$ & & $\mathbf{8 8 1 7 2 1 5 7 , 1 8}$ \\
\hline
\end{tabular}

\begin{tabular}{c|c|c|c|c|c}
\hline Código Muestra & Alófano (\%) & $\begin{array}{c}\text { Intervalos Alófano } \\
(\mathbf{\%})\end{array}$ & Área $\left(\mathbf{m}^{2}\right)$ & Potencia Alófano (m) & Volumen $\left(\mathbf{m}^{\mathbf{3}}\right)$ \\
\hline EV1-00025 & 55,93 & $54-57$ & 12505178,7 & 2,86 & 35764810,99 \\
\hline MS1-00008 & 54,21 & $54-57$ & 3404430,0 & 4,70 & 16000820,93 \\
\hline FC1-00012 & 55,51 & $54-57$ & 1584649,4 & 3,15 & 4991645,45 \\
\hline FC1-00010 & 54,72 & $54-57$ & 1221980,1 & 3,65 & 4460227,37 \\
\hline MS1-00005 & 55,44 & $54-57$ & 3190118,0 & 3,25 & 10367883,51 \\
\hline FC1-00011 & 55,00 & $54-57$ & 1409088,1 & 2,89 & 4072264,74 \\
\hline FC1-00006 & 56,74 & $54-57$ & 976297,8 & 2,56 & 2499322,26 \\
\hline EV1-00007 & 55,29 & $54-57$ & 2268295,6 & 3,65 & 8279278,91 \\
\hline EV1-00004 & 54,77 & $54-57$ & 3511729,7 & 3,40 & 11939881,09 \\
\hline TOTAL & & $\mathbf{5 4 - 5 7}$ & $\mathbf{3 0 0 7 1 7 6 7 , 3}$ & & $98376135, \mathbf{2 4}$ \\
\hline
\end{tabular}

\begin{tabular}{c|c|c|c|c|c}
\hline Código Muestra & Alófano (\%) & $\begin{array}{c}\text { Intervalos Alófano } \\
(\mathbf{\%})\end{array}$ & Área $\left(\mathbf{m}^{2}\right)$ & Potencia Alófano (m) & Volumen $\left(\mathbf{m}^{3}\right)$ \\
\hline EV1-00006 & 58,64 & $57-60$ & 3496266,8 & 3,25 & 11362867,24 \\
\hline MS1-00002 & 58,88 & $57-60$ & 721754,7 & 3,20 & 2309615,19 \\
\hline TOTAL & & $57-60$ & 4218021,6 & & 13672482,43 \\
\hline
\end{tabular}

\begin{tabular}{c|c|c|c|c|c}
\hline Código Muestra & Alófano (\%) & $\begin{array}{c}\text { Intervalos Alófano } \\
(\%)\end{array}$ & Área $\left(\mathbf{m}^{2}\right)$ & Potencia Alófano (m) & Volumen $\left(\mathbf{m}^{3}\right)$ \\
\hline FC1-00007 & 61,02 & $>60$ & 4094365,0 & 3,78 & 15476699,52 \\
\hline TOTAL & & $>60$ & 4094365,0 & & 15476699,52 \\
\hline
\end{tabular}

